\title{
Immediate Therapeutic Outcomes and Medium Term Follow up Results of Percutaneous Balloon Pulmonary Valvuloplasty in Pulmonary Valve Stenosis in the Pediatric Population
}

Dan Yin ${ }^{1}$, Xiaoyun $\mathrm{Wu}^{1}$, Ping Xiang ${ }^{1}$, Yu Zhang ${ }^{1}$, Jie Tian ${ }^{1}$, Tiewei $\mathrm{Lv}^{1}$, Qijian $\mathrm{Yi}^{1}$, and $\mathrm{Mi} \mathrm{Li}{ }^{1}$

${ }^{1}$ Chongqing Medical University Affiliated Children's Hospital

May 31, 2021

\begin{abstract}
Percutaneous balloon pulmonary valvuloplasty is a preferred treatment in pulmonary valve stenosis children with higher success rate. It is important to study the risk factors of postoperative severe pulmonary regurgitation, residual stenosis, and re-stenosis.
\end{abstract}

Immediate Therapeutic Outcomes and Medium-Term Follow-up Results of Percutaneous Balloon Pulmonary Valvuloplasty in Pulmonary Valve Stenosis in the Pediatric Population

Dan Yin ${ }^{1,4,5}$, Xiaoyun $\mathrm{Wu}^{1,2,3,4,5}$, Ping Xiang ${ }^{1,2,3,4}$, Yu Zhang ${ }^{1,4}$, Jie Tian ${ }^{1,2,3,4,5}$, Tiewei $\mathrm{Lv}^{1,2,3,4}$, Qijian $\mathrm{Yi}^{1,2,3,4,5}$, Mi Li ${ }^{1,2,3,4,5^{*}}$

\section{Authors' institutional affiliations}

Dan Yin ${ }^{1,4,5}$, Xiaoyun $\mathrm{Wu}^{1,2,3,4,5}$, Ping Xiang ${ }^{1,2,3,4}$, Yu Zhang ${ }^{1,4}$, Jie $\operatorname{Tian}^{1,2,3,4,5}$, Tiewei $\mathrm{Lv}^{1,2,3,4}$, Qijian $\mathrm{Yi}^{1,2,3,4,5}$, Mi Li ${ }^{1,2,3,4,5^{*}}$

1 Ministry of Education Key Laboratory of Child Development and Disorders, Chongqing, China,

2 National Clinical Research Center for Child Health and Disorders, Chongqing, China,

3 China International Science and Technology Cooperation Base of Child Development and Critical Disorders, Chongqing, China,

4 Department of Cardiovascular Medicine, Children's Hospital of Chongqing Medical University, Chongqing, China,

5 Chongqing Key Laboratory of Pediatrics, Chongqing, China

\section{Authors' Information}

Corresponding author: Pro. Mi Li

Department of Cardiovascular Medicine,

Children's Hospital of Chongqing Medical University,

Zhongshan Er Road, No.136, Yuzhong District,

Chongqing 400014, China

Tel: $+86-13896108463$ 
Fax: +86-023-63633496

Email: lm_dhm@163.com

\section{Key Clinical Message}

Percutaneous balloon pulmonary valvuloplasty is a preferred treatment in pulmonary valve stenosis children with higher success rate. It is important to study the risk factors of postoperative severe pulmonary regurgitation, residual stenosis, and re-stenosis.

\section{Abstract}

Background:Percutaneous balloon pulmonary valvuloplasty (PBPV) is the primary treatment for pulmonary valve stenosis (PVS).

Methods: The study consisted of 228 children with PVS who underwent PBPV from January 2004 to October 2019 at a single center. The risk factors for [?]moderate pulmonary regurgitation (PR), residual stenosis, and re-stenosis were analyzed based on the baseline patient characteristics and measured value of corresponding inspection results.

Results: Among 228 patients, follow-up results were obtained in 193 patients. The univariate analysis demonstrated that young age, low weight, small pulmonary annulus diameter, higher initial RV-PA PSEG, increased RV/systemic pressure ratio, and severe PVS were associated with [?]moderate PR. The multivariate analysis demonstrated that higher initial RV-PA PSEG and low weight were independently associated with [?]moderate PR, while higher initial RV-PA PSEG was independently associated with residual stenosis and re-stenosis.

Conclusion: PBPV is a preferred treatment in PVS children with higher success rate. Higher initial RV-PA PSEG was a significant factor for [?]moderate PR, residual stenosis, and re-stenosis.

\section{Introduction}

Pulmonary valve stenosis (PVS) is a typical congenital heart disease that represents approximately $8 \%-10 \%$ of cardiac birth defects ${ }^{[1]}$. The prevalence seems to be steadily increasing over time. Pulmonary stenosis can be valvular, sub-valvular (infundibular) or supra-valvular. Valvular stenosis is by far the most common form ${ }^{[2]}$. Percutaneous balloon pulmonary valvuloplasty (PBPV) was first reported by Kan et al. in 1982 ${ }^{[3]}$. Since then, several other studies reported successful application of this technique to treat patients with PVS. They focused on the feasibility, safety, effectiveness, and practicality of PBPV ${ }^{[4-7]}$. However, these studies were restricted to youths or adults. Thus, our study focused on a retrospective analysis of the pediatric population. In this study, the risk factors of [?]moderate pulmonary regurgitation (PR) and pulmonary re-stenosis were emphatically analyzed.

\section{Materials and Methods}

\section{Study Population}

We presented a retrospective study of 228 PVS children who underwent PBPV at the Cardiology Department of Children's Hospital of Chongqing Medical University from January 2004 to October 2019. All participants in this study were diagnosed with isolated PVS using echocardiography. PVS patients accompanied by patent foramen ovale (PFO), patent ductus arteriosus (PDA), atrial septal defects (ASDs), or ventricular septal defects (VSDs) without hemodynamic compromise were included. Patients with other complex congenital cardiac defects including double-outlet right ventricle, transposition of the great arteries, tetralogy of Fallot, and other heart diseases with hemodynamic compromise were excluded. The study protocol was approved by the Ethics Committee of Children's Hospital of Chongqing Medical University.

\section{Definition}

In this study, quantitative assessment of PVS severity was based mainly on the transpulmonary pressure 
gradient $^{[8]}$. Pulmonary stenosis severity was defined based on the 2006 ACC/AHA guidelines on the management of valvular heart disease ${ }^{[9]}$. The accompanying PR is graded as mild, moderate, or severe ${ }^{[10]}$.

\section{Data collection}

Patient and procedural data were retrospectively collected from the electronic patient database at the Pediatric Cardiology Department of Children's Hospital of Chongqing Medical University from January 2004 to October 2019. All patients completed the preoperative routine examinations, including blood routine, biochemistry, coagulation time, hepatic and renal function, arterial blood gas, 12-lead electrocardiogram (ECG), chest radiography, abdominal ultrasound, transthoracic echocardiography (TTE), cardiac catheterization, and angiography.

\section{Echocardiographic Data}

Standard M-mode and two-dimensional echocardiographic views in addition to color Doppler and continuouswave Doppler were carried out to examine the velocity flow and morphology of the pulmonary valve, peak PVS gradient, right ventricular dimensions, regurgitation of tricuspid and pulmonary valve in addition to its degree, and systolic and diastolic functions of the left ventricular. Using the simplified Bernoulli equation $\left({ }^{P}=\right.$ $4 v 2$, themaximumpeakinstantaneoussystolicpressuregradientwasestimatedfromthetranspulmonary flowvelocitycurve.

\section{Cardiac catheterization and angiography}

Venous access was usually achieved via the femoral vein and where the pig tail or balloon floating catheter is inserted for right cardiac catheterization. All procedures were performed with a single balloon. The data acquired from initial PBPV cardiac catheterization and postoperative reports included right ventricular (RV) systolic pressure, pulmonary artery (PA) systolic pressure, RV-PA PSEG, and systemic systolic blood pressure.

\section{Statistical Analysis}

All statistical tests were performed using SPSS version 23.0. Categorical variables are expressed as frequency with percentage, while continuous variables are expressed as mean and median with interquartile range (IQR). The chi-square or Fisher's exact test was used to compare the categorical variables. The Wilcoxon rank sum test was used to compare the continuous variables between different groups. Logistic regression analysis was performed to identify the independent risk factors of PR severity and re-stenosis while adjusting for potential confounding variables. The odds ratios (ORs) and $95 \%$ confidence intervals (CI) were obtained. Freedom from repeat PBPV or surgical intervention was evaluated using the Kaplan-Meier curve. Statistical significance was defined as a $\mathrm{P}$ value of $<0.05$.

\section{Results}

\section{Patients' Baseline Characteristics}

Among the 228 participants, except for 35 patients without follow-up data, the remaining 193 patients had a mean age at initial PBPV of 2.56 years (median: 1.58 years, IQR: 11 months- 3.13 years, range: 21 days14.25 years), mean weight at initial PBPV of $12.80 \mathrm{~kg}$ (median: $11 \mathrm{~kg}$, IQR: 9-15 kg, range: 2.24-55 kg), mean height at initial BPV of $79.21 \mathrm{~cm}$ (median: $75 \mathrm{~cm}$, IQR: $68.75-84 \mathrm{~cm}$, range: $46-159 \mathrm{~cm}$ ), mean BSA at initial BPV of $0.474 \mathrm{~m}^{2}$ (median: $0.437 \mathrm{~m}^{2}$, IQR: $0.384-0.485 \mathrm{~m}^{2}$, range: $0.162-1.376 \mathrm{~m}^{2}$ ), mean initial peak echocardiographic PVS gradient of $60.66 \mathrm{mmHg}$ (median: $53 \mathrm{mmHg}$, IQR: 41.99-73.62), and mean initial RV-PA PSEG of $54.58 \mathrm{mmHg}$ (median: $49 \mathrm{mmHg}$, IQR: 33-66.25). The mean RV-PA PSEG postvalvuloplasty was $19.19 \mathrm{mmHg}$ (median: $14 \mathrm{mmHg}$, IQR: 9-22.5 mmHg), mean initial $\mathrm{RV} /$ systemic pressure ratio was 0.82 (median: 0.71, IQR: $0.60-0.94$ ), and mean $\mathrm{RV} /$ systemic pressure ratio post-valvuloplasty was 0.47 (median: 0.42, IQR: 0.35-0.52). The mean balloon/annulus ratio was 1.23 (median: 1.20, IQR: 1.201.25). The average pulmonary annulus diameter was $12.35 \mathrm{~mm}$ (median: $12.3 \mathrm{~mm}$, IQR: $10.2 \mathrm{~mm}-14.08$ $\mathrm{mm}$ ) measured by angiography. (Table 1, Figure 1).

Immediate results of percutaneous balloon pulmonary valvuloplasty 
All 193 patients with follow-up data had preoperative and postoperative cardiac catheterization results. Immediately after PBPV, the right ventricular (RV) systolic pressures reduced significantly from 77.23 mmHg (median: $71 \mathrm{mmHg}$, IQR: $56-91 \mathrm{mmHg}$ ) to $43.81 \mathrm{mmHg}$ (median: $40 \mathrm{mmHg}$, IQR: $33.5-48 \mathrm{mmHg}$ ), the pulmonary artery (PA) systolic pressure increased from $22.40 \mathrm{mmHg}$ (median: $22 \mathrm{mmHg}$, IQR: 19-26 $\mathrm{mmHg}$ ) to $24.27 \mathrm{mmHg}$ (median: $24 \mathrm{mmHg}$, IQR: 21-27 mmHg), and RV-PA PSEG reduced significantly from $54.84 \mathrm{mmHg}$ (median: $49 \mathrm{mmHg}$, IQR: $33-67 \mathrm{mmHg}$ ) to $19.50 \mathrm{mmHg}$ (median: $14 \mathrm{mmHg}$, IQR: 9-23 $\mathrm{mmHg}$ ) (Table 2). Immediate success was defined as a pulmonary gradient of $<40 \mathrm{mmHg}$ after PBPV, and the immediate success rate was $89.6 \%$.

\section{Follow-up}

\section{Echocardiographic results}

In most patients with recent echocardiogram reports, 186 (96.4\%) had PR and 7 (3.6\%) were free from PR. None of the patients had absence of tricuspid regurgitation (TR), $136(70.5 \%)$ had mild TR, $51(26.4 \%)$ had mild to moderate TR, $4(2.1 \%)$ had moderate TR, $1(0.5 \%)$ had moderate to severe TR, and $1(0.5 \%)$ had severe TR. Nearly half of the cohort (48.7\%) had RV dilation, $99(51.3 \%)$ had normal RV size on most recent echocardiogram reports, some had significant right ventricular enlargement at the beginning, but the extent of the enlargement gradually diminished during follow-up. In follow-up, the pulmonary transvalvular peak gradients were dropped obviously. (Table 3 ).

\section{Risk factors for [?]moderate pulmonary regurgitation}

Among the 193 patients who had follow-up echocardiography results, PR occurred in 186 patients, including 110 with mild PR and 76 with moderate to severe PR.

Univariate analysis results demonstrated that patients with [?]moderate PR during follow-up had a younger age, shorter height, lower weight, and smaller body surface area at the time of initial PBPV $(\mathrm{P}<0.05)$. Moreover, patients in this group were inclined to have a smaller pulmonary annulus diameter at baseline, higher RV-PA PSEG, and increased RV/systemic pressure ratio $(\mathrm{P}<0.05)$. Severe PVS will more likely result in [?]moderate PR $(\mathrm{P}<0.05)$, while no significant difference was observed between the balloon and annulus ratio (1.20 (IQR: $1.20-1.25)$ and 1.24 (IQR: 1.20-1.30), $\mathrm{P}>0.05$ ). Of the 76 patients with [?]moderate PR, $11(14.5 \%)$ presented with pulmonary artery valve dysplasia or abnormal valve morphology, of whom 7 had pulmonary valves with two lobes and 4 had pulmonary valves with dysplasia. (Table 4 ).

Multivariate analysis results illustrated that low weight (OR: 0.668, 95\% CI: 0.518-0.862) and higher initial RV-PA PSEG (OR: 1.017, 95\% CI: 1.003-1.031) were independent risk factors for [?]moderate PR (P <0.05) (Table 5).

\section{Residual stenosis and re-stenosis during follow-up}

Among 193 patients with follow-up data, 32 (16.6\%) had residual stenosis following initial PBPV or restenosis during follow-up, of whom 9 required re-intervention. The other 9 children underwent re-intervention including 7 who underwent repeat PBPV at an average of 2.12 years (range: 6 months to 5 years) after initial $\mathrm{PBPV}$, and 2 children underwent surgical intervention at 6 months and 1.33 years after initial PBPV (Figure 2).

\section{Risk factors for residual stenosis or re-stenosis}

All study participants with follow-up echocardiography were classified into two groups. The first group (83.4\%) included patients with a residual stenosis of $<40 \mathrm{mmHg}$ after initial PBPV. The other group (16.6\%) included patients with residual stenosis after initial PBPV or re-stenosis of [?]40 mmHg during follow-up, and some of them underwent repeat intervention.

Univariate analysis results showed that the group with residual stenosis or re-stenosis had younger age, lower weight, and smaller body surface area at the time of initial PBPV. Moreover, patients in that group had small pulmonary annulus diameter at baseline, higher RV-PA PSEG, and increased RV/systemic pressure 
ratio $(\mathrm{P}<0.05)$. Critical PVS was statistically associated with residual stenosis or repeat intervention, while BAR had no significant association. (Table 6).

Multivariate analysis results showed that higher initial RV-PA PSEG (OR: 1.056, 95\% CI: 1.035-1.078) was a significant independent risk factor for residual stenosis after initial PBPV or re-stenosis of [?]40 $\mathrm{mmHg}$ during follow-up $(\mathrm{P}<0.05)$ (Table 7).

\section{Discussion}

Since $1982^{[3]}$, PBPV is a primary technique that has been extensively used for PVS treatment. Several studies have discussed the safety, effectiveness, feasibility, and practicality of this technique. We performed a retrospective review of all pediatric population definitively diagnosed with PVS, and all study participants underwent PBPV at our institution. This is one of the largest series to describe the outcomes of the pediatric population diagnosed with PVS after PBPV.

Several studies reported the immediate, short-, intermediate-, and long-term effectiveness and safety of PBPV in different centers ${ }^{[11,12]}$. A previous study conducted by Dian Hong et al. ${ }^{[13]}$ reported a restenosis rate of $6.4 \%$ (10 of 158) and reintervention rate of 3.7\% (6 of 158), and indicated that PBPV was the primary treatment as it was safe and minimally invasive. Recently, Hansen et al. ${ }^{[14]}$ conducted a multicenter study with the longest follow-up duration of up to 25 years, in this study, $83 \%$ of the study patients $(\mathrm{n}=207)$ had a significant decrease in PVS after undergoing initial PBPV, while 17\% required repeat intervention.

The adverse reactions caused by chronic PR were unclear. In a previous study conducted by Merino-Ingelmo et al.[15], which included 53 patients who underwent PBPV, the majority of patients did not experience PR before the procedure; however, none of the patients showed absence of PR at the end of the study. In a larger study, David M. Harrild et al. ${ }^{[16]}$ showed that severe PR and mild RV dilation occurred after PBPV. In this study, $34 \%$ of patients had a PR fraction of $>15 \%$, and $40 \%$ had an RV end-diastolic volume z-score of [?]2. However, severe PR or RV dilation rarely occurred. In the latest multicenter research by Hansen et al. ${ }^{[14]}$, which included a larger sample size $(\mathrm{n}=248), 42 \%(\mathrm{n}=33)$ of them had [?]moderate PR. This study indicated that young age, low weight, higher baseline RV-PA PSEG, increased baseline RV/systemic pressure ratio, and critical PVS were the risk factors for higher PR.

Our research showed that $76(39.4 \%)$ of the follow-up patients had [?]moderate PR. In the multivariate analysis, lower weight and higher initial RV-PA PSEG were independent risk factors for [?]moderate PR. Although the degree of PR was increasing, it was well tolerated, and none of our study patients underwent pulmonary valve replacement for severe PR.

Several studies have been conducted to determine the target BAR. In previous studies, the finding that significant PR was uncommon in patients with a BAR of $<1.4$ support the appropriateness of 1.2 to 1.4 as a target BAR range. In a study by David M. Harrild et al. ${ }^{[16]}$, patients with a smaller pulmonary valve z-score were more likely treated with a larger BAR. However, the results showed that using a BAR of $>1.4$ was more likely to cause severe PR. Sagar J. Pathak et al. ${ }^{[17]}$ suggested that using a BAR of $<1.2$ significantly decreased the PR rate than a BAR of $>1.2$ without affecting the procedure efficacy and increasing the need for repeat intervention for residual PVS. A latest study by Dian Hong et al. ${ }^{[13]}$ showed that the choice of BAR could affect the immediate therapeutic effects and medium-term follow-up results. The BAR they selected was 1.2-1.4, which was widely accepted and has already been widely recommended in some guidelines. Our study showed that BAR was not a risk factor for [?]moderate PR.

Some limitations exist in the present study. This study had a short follow-up duration, was retrospective in nature, and was conducted at a single center. This study had a relatively short follow-up period after initial PBPV, and the maximum follow-up time was no more than 9 years. The participants were from a single center rather than a multicenter, and a data bias may exist. Due to the retrospective cohort design of this study, the collection of clinical data was inadequate. Notwithstanding, this research can still be used as a reference for future studies. Therefore, with these limitations, a more comprehensive assessment is required for future work. 


\section{Conclusions}

PBPV is a definitive therapy in the pediatric population with PVS based on its safety, effectiveness, feasibility, and practicality. Although PBPV was effective in relieving PVS with low risk of residual stenosis and restenosis, there was considerable risk of moderate and severe PR in the late follow-up. Although PBPV was efficient in alleviating PVS with lower risk of residual stenosis and restenosis, there was still a significant risk of [?]moderate PR in the late follow-up. Furthermore, PR may be aggravated with time; hence, further studies are essential to explore mechanism underlying the development of PR.

\section{List of abbreviations}

PBPV: Percutaneous balloon pulmonary valvuloplasty

PVS: Pulmonary valve stenosis

RV: Right ventricular

PA: Pulmonary artery

PSEG: Peak systolic ejection gradient

PR: Pulmonary regurgitation

TR: Tricuspid regurgitation

PFOs: Patent foramen ovale

PDAs: Patent ductus arteriosus

ASDs: Atrial septal defects

VSDs: Ventricular septal defects

BAR: Balloon/annulus ratio

BSA: Body surface area

IQR: Interquartile range

ECG: Electrocardiography

TTE: Transthoracic echocardiography

ACC: American College of Cardiology

AHA: American Heart Association

Ethics approval and consent to participate

The study protocol was approved by the Ethics Committee of Children's Hospital of Chongqing Medical University.

\section{Consent for publication}

Consent for publication.

\section{Availability of data and materials}

The processed data and material used to support the findings of this study are included within the article and they are can be accessed.

\section{Funding}

This study did not receive any financial support from external sources.

\section{Competing interests}


The authors of this article have no conflict of interest with other products or organizations.

\section{Authors' contributions}

Dan Yin: conception and design, acquisition of data, analysis and interpretation of data, drafting of the article, and manuscript revision

Xiaoyun Wu: conception and design and manuscript revision

Ping Xiang: conception and design and acquisition of data

Yu Zhang: acquisition of data

Jie Tian: analysis and interpretation of data

Tiewei LV: conception and design and manuscript revision

Qijian Yi: conception and design

Mi Li: conception and design and final approval of the version to be published

\section{Acknowledgements}

Not applicable

\section{References}

[1] Denise van der Linde, Konings Elisabeth-EM, Slager Maarten-A, et al. Birth prevalence of congenital heart disease worldwide: a systematic review and meta-analysis[J]. Journal of the American College of Cardiology, 2011, 58(21): 2241-2247.

[2] Judith-Anne-Adriane-Ellen Cuypers, Witsenburg Maarten, van der Linde Denise, et al. Pulmonary stenosis: update on diagnosis and therapeutic options[J]. Heart, 2013, 99(5): 339-347.

[3] Jean-S Kan, White Jr Robert-I, Mitchell Sally-E, et al. Percutaneous balloon valvuloplasty: a new method for treating congenital pulmonary-valve stenosis[J]. New England Journal of Medicine, 1982, 307(9): 540-542.

[4] Brian-W McCrindle. Independent predictors of long-term results after balloon pulmonary valvuloplasty. Valvuloplasty and Angioplasty of Congenital Anomalies (VACA) Registry Investigators.[J]. Circulation, 1994, 89(4): 1751-1759.

[5] Weryński P, Rudziński A, Król-Jawień W, et al. Percutaneous balloon valvuloplasty for the treatment of pulmonary valve stenosis in children-a single centre experience[J]. Kardiologia Polska, 2009, 67(4): 369-375.

[6] Rajiv Devanagondi, Peck Dan, Sagi Janaki, et al. Long-term outcomes of balloon valvuloplasty for isolated pulmonary valve stenosis[J]. Pediatric Cardiology, 2017, 38(2): 247-254.

[7] Varun Aggarwal, Mulukutla Venkatachalam, Maskatia Shiraz, et al. Outcomes after Balloon Pulmonary Valvuloplasty for Critical Pulmonary Stenosis and Incidence of Coronary Artery Fistulas[J]. The American Journal of Cardiology, 2018, 121(12): 1617-1623.

[8] Helmut Baumgartner, Hung Judy, Bermejo Javier, et al. Echocardiographic assessment of valve stenosis: EAE/ASE recommendations for clinical practice[J]. Journal of the American Society of Echocardiography, 2009, 22(1): 1-23.

[9] Robert-O Bonow, Carabello Blase-A, Chatterjee Kanu, et al. ACC/AHA 2006 guidelines for the management of patients with valvular heart disease: a report of the American College of Cardiology/American Heart Association Task Force on Practice Guidelines (writing Committee to Revise the 1998 guidelines for the management of patients with valvular heart disease) developed in collaboration with the Society of Cardiovascular Anesthesiologists endorsed by the Society for Cardiovascular Angiography and Interventions and the Society of Thoracic Surgeons[J]. Journal of the American College of Cardiology, 2006, 48(3): e1-e148. 
[10] P-S Rao, Galal O, Patnana Madhavi, et al. Results of three to 10 year follow up of balloon dilatation of the pulmonary valve[J]. Heart, 1998, 80(6): 591-595.

[11] John-J Parent, Ross Michael-M, Bendaly Edgard-A, et al. Results of pulmonary balloon valvuloplasty persist and improve at late follow-up in isolated pulmonary valve stenosis[J]. Cardiology in the Young, 2017, 27(8): 1566-1570.

[12] Petra Loureiro, Cardoso Barbara, Gomes Inês-B, et al. Long-term results of percutaneous balloon valvuloplasty in neonatal critical pulmonary valve stenosis: a 20-year, single-centre experience[J]. Cardiology in the Young, 2017, 27(7): 1314-1322.

[13] Dian Hong, Qian Ming-Yang, Zhang Zhi-Wei, et al. Immediate Therapeutic Outcomes and Medium-term Follow-up of Percutaneous Balloon Pulmonary Valvuloplasty in Infants with Pulmonary Valve Stenosis: A Single-center Retrospective Study[J]. Chinese Medical Journal, 2017, 130(23): 2785.

[14] Hansen RL, Naimi I, Wang H, et al. Long-term outcomes up to 25 years following balloon pulmonary valvuloplasty: A multicenter study[J]. Congenital Heart Disease, 2019, 14(6): 1037-1045.

[15] Raquel Merino-Ingelmo, Santos-de Soto Jose, Coserria-Sanchez Felix, et al. Long-term results of percutaneous balloon valvuloplasty in pulmonary valve stenosis in the pediatric population[J]. Revista Espanola de Cardiologia (English Edition), 2014, 67(5): 374-379.

[16] David-M Harrild, Powell Andrew-J, Trang Trang-X, et al. Long-term pulmonary regurgitation following balloon valvuloplasty for pulmonary stenosis: risk factors and relationship to exercise capacity and ventricular volume and function[J]. Journal of the American College of Cardiology, 2010, 55(10): 1041-1047.

[17] Sagar-J Pathak, Pockett Charissa-R, Moore John-W, et al. Effect of balloon: annulus ratio on incidence of pulmonary insufficiency following Valvuloplasty[J]. Congenital Heart Disease, 2016, 11(5): 415-419.

Figure 1: Flow diagram of PBPV patient outcomes

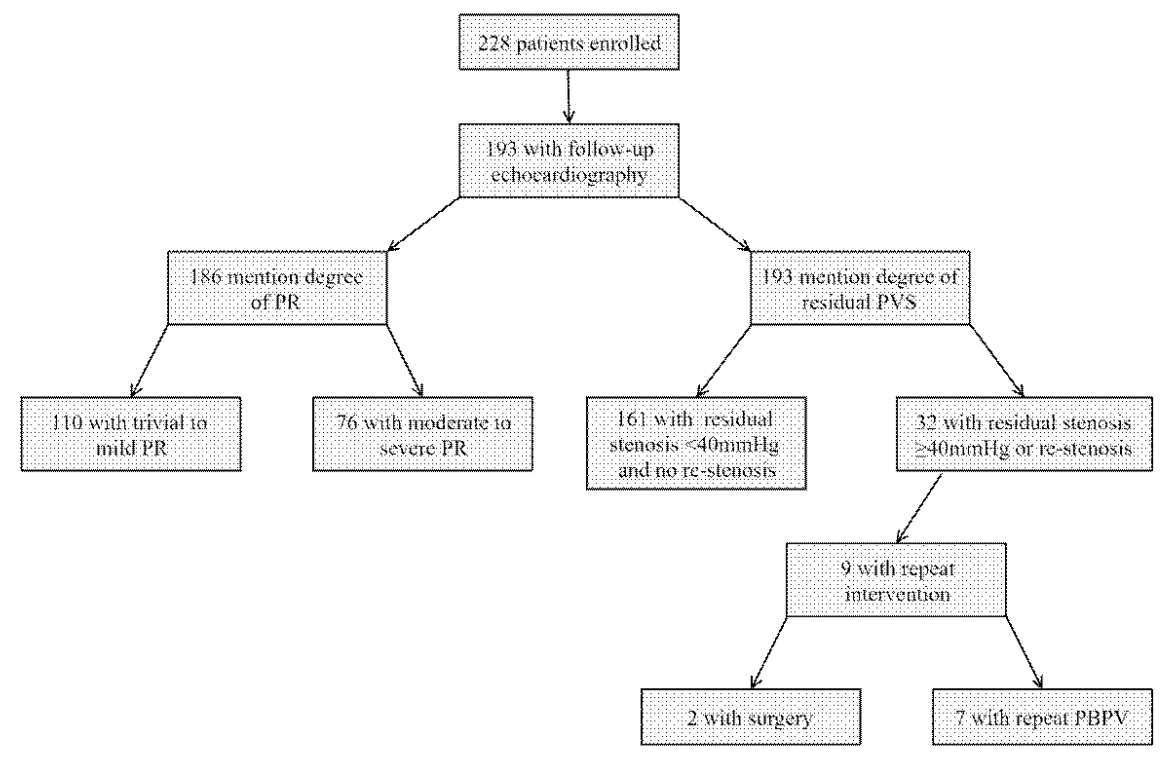

Notes: Of 228 patients, 193 had available follow-up echocardiography results, 32 had residual PVS of $\geq 40 \mathrm{~mm} \mathrm{Hg}, 9$ had repeat intervention (7 had repeat PBPV; 2 had surgery), 76 had $\geq$ moderate $P R$, and 0 had pulmonary valve replacement. 
Figure 2: Kaplan-Meier curve demonstrating freedom from repeat intervention. Repeat intervention included repeat PBPV, surgery for residual PVS, or pulmonary valve replacement for $\mathrm{PR}$

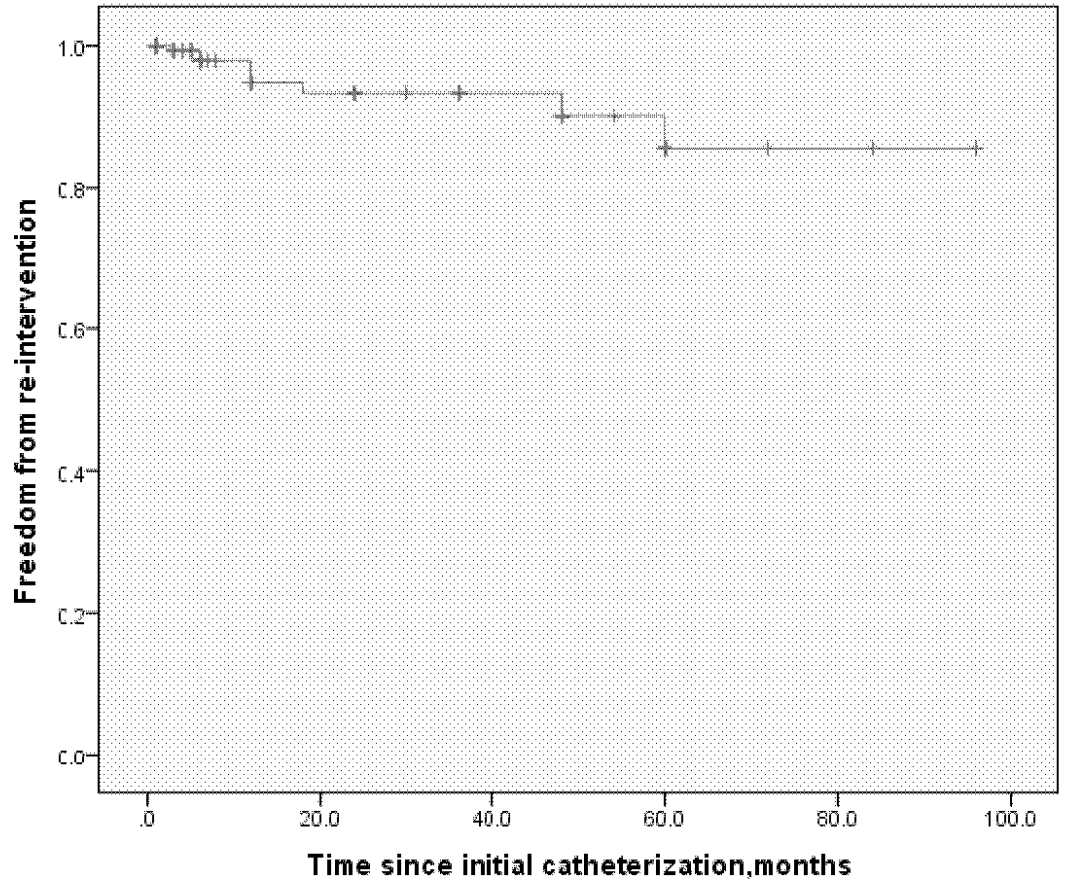

